\title{
Changes in urine headspace composition as an effect of strenuous walking
}

\author{
Devasena Samudrala $^{1}$ - Brigitte Geurts ${ }^{2}$ Phil A. Brown ${ }^{1,3}$ - Ewa Szymańska ${ }^{2,3}$. \\ Julien Mandon ${ }^{1} \cdot$ Jeroen Jansen $^{2} \cdot$ Lutgarde Buydens $^{2} \cdot$ Frans J. M. Harren ${ }^{1}$ • \\ Simona M. Cristescu ${ }^{1}$
}

Received: 9 February 2015/ Accepted: 20 May 2015/Published online: 31 May 2015

(c) The Author(s) 2015. This article is published with open access at Springerlink.com

\begin{abstract}
The present investigation uses proton transfer reaction mass spectrometry (PTR-MS) combined with multivariate and univariate statistical analyses to study potential biomarkers for altered metabolism in urine due to strenuous walking. Urine samples, in concurrence with breath and blood samples, were taken from 51 participants (23 controls, 11 type-1 diabetes, 17 type- 2 diabetes) during the Dutch endurance walking event, the International Four Days Marches. Multivariate analysis allowed for discrimination of before and after exercise for all three groups (control, type-1 and type-2 diabetes) and on three out of 4 days. The analysis highlighted 12 molecular ions contributing to this discrimination. Of these, acetic acid in urine is identified as a significant marker for exercise effects induced by walking; an increase is observed as an effect of walking. Analysis of acetone concentration with univariate tools resulted in different information when compared to breath as a function of exercise, revealing an interesting effect of time over the 4 days. In breath, acetone provides an immediate snapshot of metabolism, whereas
\end{abstract}

Electronic supplementary material The online version of this article (doi:10.1007/s11306-015-0813-8) contains supplementary material, which is available to authorized users.

Simona M. Cristescu

s.cristescu@science.ru.nl

1 Institute Molecules \& Materials, Life Science Trace Gas Facility, Radboud University Nijmegen, 6500 GL Nijmegen, The Netherlands

2 Institute Molecules \& Materials, Department Analytical Chemistry, Radboud University Nijmegen, 6500 GL Nijmegen, The Netherlands

3 TI-COAST, Science Park 904, 1098 XH Amsterdam, The Netherlands urinary acetone will result from longer term diffusion processes, providing a time averaged view of metabolism. The potential to use PTR-MS measurements of urine to monitor exercise effects is exhibited, and may be utilized to monitor subjects in mass participation exercise events.

Keywords Urine - Walking · Acetic acid · PTR-MS . Acetone

\section{Introduction}

Due to a persistent absence of phenotypic symptoms during progression, many chronic diseases like diabetes can remain undiagnosed during the early stages of development. Even after diagnosis, the suitability and effectiveness of medication or lifestyle interventions (diet, exercise etc.) are difficult to interpret, yet critically important for managing the treatment of a chronic disease. Personal omics profiling (POP) aims to tackle these challenges by scanning individuals in detail to identify the genetic basis for disease risk or treatment efficacy, and using this knowledge to enable daily monitoring with post-genomic technology (Chen et al. 2012; Roukos 2008). Large-scale implementation of this approach would however put a disproportionate burden on healthcare resources, as this requires extensive use of highly advanced technology. Likewise, this would require considerable effort of the healthy examinee, as he/she would need to regularly visit the appropriate medical infrastructure for a relatively invasive procedure; typically the provision of blood samples. Although highly sensitive to detect and monitor a broad range of potential diseases, in its current state POP is too impractical to be viably implemented.

A truly feasible implementation would be to collect samples in locations where many members of the target 
group are voluntarily present. Several samples (breath, urine) can then be obtained considerably less-invasively, without requirement for specifically trained personnel. However, metabolomics technology is put to the challenge for such scenarios, as the sheer number of samples generated on such highly populated locations requires rapid analysis and the diluted state of specifically volatile samples requires highly sensitive analysis platforms.

The volatile organic compounds (VOCs) emitted from the skin (Turner et al. 2008), exhaled in breath (Buszewski et al. 2007; Schwarz et al. 2009; Lourenço and Turner 2014) or present in urine (Huang et al. 2013; Mochalski et al. 2012) have been well characterized in healthy people in the literature (Costello et al. 2014). Changes in the concentration of specific VOCs can indicate particular diseases (Smolinska et al. 2014) and changes in metabolic state, such as nitric oxide in airway inflammation (Barnes et al. 2010) or acetone for diabetes (Storer et al. 2011), and as such they are considered biomarkers (Mazzatenta et al. 2013). Proton transfer reaction mass spectrometry (PTRMS) (Lindinger et al. 1998; Blake et al. 2009) is a popular, highly sensitive, on-line tool used to measure concentrations of excreted metabolites, particularly in breath (Herbig et al. 2009; Schwarz et al. 2009) but also urine (Pinggera et al. 2005). The technique allows rapid analysis of VOCs, due to its online capability. Accurate measurements of VOC concentrations are possible in seconds. The present study aims to use PTR-MS coupled with multivariate and univariate statistical techniques to investigate potential biomarkers for exercise altered metabolism in urine. In a previous publication, breath acetone, which was measured with proton transfer reaction ion trap mass spectrometry, correlated positively with both non-esterified fatty acids and beta-hydroxybutyrate (BOHB) (markers in blood for fatty acid metabolism), providing real-time information on fat burning (Samudrala et al. 2014). We now examine the relation between urine acetone and breath acetone and evaluate the VOCs in urine as possible biomarkers for the effect of 4 days strenuous walking, as well as their interplay with medicated type 1 and type 2 diabetes mellitus.

\section{Materials and methods}

\subsection{Subjects}

All subjects participated in the International Four Days Marches, July 2012, an annual walking event in Nijmegen, the Netherlands, organized by the Dutch Walking Organization (KNBLO-NL). In total 51 participants gave urine samples. Among them 23 were control (CT), 11 type-1 diabetes mellitus (T1DM) and 17 type- 2 diabetes mellitus (T2DM). Participants included 28 males and 23 females with an age range of 25-85 years. Depending on their age and gender, participants walked 30,40 or $50 \mathrm{~km}$ per day. The details of the subjects who participated in this study are shown elsewhere (Samudrala et al. 2014). This study was approved by the Medical Ethical Committee of the Radboud University Nijmegen Medical Centre. Informed consent was obtained from all individual participants included in the study, and the study was conducted in accordance with the Declaration of Helsinki.

\subsection{Urine samples}

Urine samples were collected twice a day for 4 consecutive days from each subject, once in the morning, prior to walking (between 3:30 am and 8:30 am) and another at the end of the walk (between 10:30 am and 17:30 pm).

After collection, the samples were transported via a cooler $\left(\sim 6^{\circ} \mathrm{C}\right)$ to a storage freezer of temperature $-80^{\circ} \mathrm{C}$. Samples were without any centrifuge separation and without any anti-bacterial additives. The samples were later defrosted, separated into two vials and refrozen. One vial was used for VOCs analysis with PTR-MS and the other was used for creatinine measurements. Creatinine was measured via an enzymatic method using an ARCHITECT clinical chemistry analyzer (Abbott Laboratories, Abbott Park, Illinois, USA).

\subsection{VOCs analysis from urine headspace}

Urine measurements were performed with an in-house built proton transfer reaction mass spectrometer (PTR-MS). A detailed description of this instrument has been given elsewhere (Steeghs et al. 2004) and the instrument bears a strong similarity to commercially available devices (Lindinger et al. 1998). Here, we give only a brief introduction to the technique. Proton transfer reaction is a soft ionization method in which VOCs with molecular mass (M) are ionized by the transfer of a proton from a hydronium ion $\left(\mathrm{H}_{3} \mathrm{O}^{+}\right)$, and are detected at mass $\mathrm{M}+1$ using a quadrupole mass spectrometer. Signal intensity of the hydronium ion and the protonated water cluster $(\mathrm{m} / \mathrm{z}, 19$ and $\mathrm{m} / \mathrm{z}, 37)$ are typically measured for normalization. Apart from them, 38 other ions were measured, including acetone $(\mathrm{m} / \mathrm{z}, 59)$; the dwell time for each ion was $0.2 \mathrm{~s}$.

Samples to be measured by PTR-MS were removed from the freezer the night before the measurements to defrost at room temperature $\left(\sim 20{ }^{\circ} \mathrm{C}\right)$. The following day the instrument was calibrated and samples prepared and measured. $10 \mathrm{ml}$ of urine was transferred from the vial to a glass cuvette with a $40 \mathrm{ml}$ available volume. The closing lid of the glass cuvette was sealed in place with a metal fastening clip and an O-ring, with one gas inlet and one gas outlet port to allow sampling. A constant flow of $2 \mathrm{l} / \mathrm{h}$ of 
air was passing through the cuvette to flush the head space. The outlet for this flow was side sampled by the PTR-MS at $1.5 \mathrm{l} / \mathrm{h}$. For the duration of the measurement the samples remained at room temperature. The intensity of the VOC signal was observed to decrease with time, due to the continuous flushing of the headspace. Therefore, to prevent the concentration declining, the headspace was flushed and measured for 4 min only for each sample. Beyond this time the headspace concentration drop becomes unacceptable (more than $18 \%$ drop). Three samples were measured sequentially using an automatic valve system (van Dam et al. 2012) to switch between each cuvette. An example of this measurement is shown in supplementary material: S1, with a repetitious measurement for method validation. The product ion intensities, measured in counts per seconds (cps) were normalized to reagent ion signal, which gave intensities in normalized counts per second (ncps). These were further normalized to creatinine concentrations (mmol) to give final units of ncps $\mathrm{mmol}^{-1}$. This allowed the detected signals to be normalized with respect to the level of biological dilution present in the sample (Amann and Smith 2013). For the duration of analysis, the transfer lines from the valves to the PTR-MS were heated up to $55{ }^{\circ} \mathrm{C}$ to prevent condensation.

The consistency of the analysis was checked by calibrating each day, before the start of the experiment with a standard gas mixture consisting of methanol $(\mathrm{m} / \mathrm{z} 33)$, acetaldehyde $(\mathrm{m} / \mathrm{z}, 45)$, acetone $(\mathrm{m} / \mathrm{z}, 59)$, isoprene $(\mathrm{m} / \mathrm{z} 69)$, benzene $(\mathrm{m} / \mathrm{z}, 79)$, toluene $(\mathrm{m} / \mathrm{z}, 93), O$-xylene $(\mathrm{m} / \mathrm{z}, 107)$ and alpha-pinene $(\mathrm{m} / z$ 137), each in $1000 \mathrm{ppbv} \pm 5 \%$ (ppbv $=$ part per billion volume $)$ in a nitrogen dilution gas (Linde, Dieren, the Netherlands). The calibration was performed using different concentrations, from 35 to $1000 \mathrm{ppbv}$ obtained by dilution of the standard mixture with nitrogen gas.

\subsection{Statistical analysis}

The data was first analyzed using a multivariate technique allowing the significance of all ions to be assessed. Univariate analysis was then repeated for those ions of highest significance to the multivariate model. Finally, the $\mathrm{m} / \mathrm{z} 59$ product ion of acetone was analyzed univariately for comparison with breath. The methods of univariate and multivariate analysis used in this analysis are explained below.

\subsection{Multivariate analysis}

Analysis with multilevel partial least squares discriminate analysis (M-PLS-DA) (van Velzen et al. 2008; Szymańska et al. 2012) was carried out to study the effects of exercise from a multivariate perspective. Variation in the data contains an inter-individual part and an intra-individual part. The inter-individual subject variation describes the difference between the subjects, which is unrelated to the effect of exercise. In this study each subject participated both before and after exercise (and therefore serves as his/ her own control), allowing for an intra-subject evaluation of the effect of exercise. In order to study the effect of exercise on the different groups, a separate M-PLS-DA model was built for each of the three groups (T1DM, T2DM and CT). The data were auto-scaled before analysis by M-PLS-DA. To evaluate the exercise effect on the different days, separate M-PLS-DA models were built for each of the days, taking all the groups together. The models aim to discriminate between before and after walking, and allow for identification of those ions that are important for this classification. Thirty-three ion signals in the range of $m / z 33-m / z 117$ were chosen for this analysis from the forty that were measured. Five ions were discarded because their count rate fell consistently below the detection limit of the instrument.

As a measure of model performance, the area under the receiver operator curve (AUROC) was calculated. The AUROC represents a ratio between the number of true positives and the number of false positives in the classification, where an AUROC of 1 represents perfect classification (Zweig and Campbell 1993). All M-PLS-DA models included double cross validation (van Velzen et al. 2008; Smit et al. 2007; Westerhuis et al. 2008). The optimal number of latent variables was chosen with a fourfold single cross validation. A subsequent fivefold double cross validation was used to assess the model performance on a test set. The statistical significance of the model performance was assessed by a subsequent permutation analysis with 1000 realizations. To evaluate which ions played an important role in this classification, the rank products of the variables in the model are used. All variables were ranked according to their PLS regression coefficients. The variables with the lowest rank products are the ones with the largest discriminative capability. The significance of the ranks was assessed by comparing the variable ranks of the model to those in the 1000 permutation tests, and a $p$ value was assigned accordingly. Results are represented in a radar plot, where each variable is presented on one of the radial axes (Saary 2008). Against each axis, the value $1-p$ is plotted, such that tests with lower $p$-values are positioned toward the outside of the radar field, and higher $p$-values are positioned toward the middle of the graph.

\subsection{Univariate analysis}

The normality of the data distribution was evaluated using a Lillefors test. All the ion signals measured from the urine headspace were found to have a non-normal distribution, 
such that non-parametric tests were used for all subsequent analyses. A Wilcoxon signed rank test was used to determine the effect of exercise on each individual mass. To evaluate the effect of time-the change of concentration over the 4 days - the Friedman test was used because of its ability to handle multiple attempts. Both the Wilcoxon signed-rank test and the Friedman test are 'paired' tests, comparing data from the same subject over different settings. Associations between the intensity of two ion species were evaluated using Spearman correlations. Any $p$-values $<0.05$ were considered significant and $p<0.1$ were considered trend to be significant. All statistical analyses were performed in MATLAB (version 2014a, The Mathworks, Natick, Massachusetts, USA). Boxplots were made in Origin (version 9.0, Origin lab, Northampton, Massachusetts, USA).

\section{Results}

First, the effect of exercise on the detected VOCs is shown and studied as a whole using multivariate analysis. After that, possible identification and univariate analysis of significant ions is evidenced. Finally, the effect of exercise on headspace acetone is evaluated by univariate analysis.

\subsection{Multivariate analysis of the full data-set}

Multivariate analysis was used to study the effect of exercise on detected ions and consequently emitted compounds from the urine headspace. A different classification model to extract the effect of exercise was made for each of the three groups, and the model performances are shown in Table 1(a).

In all three groups, M-PLS-DA is able to extract a higher significant exercise effect than classification of a randomly permuted dataset. The highest model performance is achieved for the CT group, followed by T2DM, as can be seen by comparing the AUROC in Table 1(a). The contribution of each mass $(\mathrm{m} / \mathrm{z}$ value) to the classification model is represented in a radar plot in Fig. 1. All three

Table 1 The ability of the models used to distinguish between before and after exercise is shown separately for (a) each group, for all 4 days together, and for (b) each day, for all three groups together

\begin{tabular}{|c|c|c|c|c|c|}
\hline \multicolumn{3}{|c|}{ (a) Model performance per group } & \multicolumn{3}{|c|}{ (b) Model performance per day } \\
\hline Group & AUROC & $p$ value & Day & AUROC & $p$ value \\
\hline CT & 0.87 & $<0.001$ & 1 & 0.63 & 0.012 \\
\hline T1DM & 0.70 & 0.004 & 2 & 0.64 & 0.066 \\
\hline \multirow[t]{2}{*}{ T2DM } & 0.81 & $<0.001$ & 3 & 0.90 & $<0.001$ \\
\hline & & & 4 & 0.94 & $<0.001$ \\
\hline
\end{tabular}

groups are represented in the same figure, allowing direct comparison of the variable's importance to each of the models generated from a particular group. The outermost, bold black circle of the graph indicates the $5 \%$ significance level. Values on and outside of this circle are considered significant. The circle drawn with a thinner line indicates the $10 \%$ significance level. Ions near to the circumferences of these two circles are of particular importance.

Considering the 33 ions involved in this analysis, three ions: $m / z 61, m / z, 62$ and $m / z, 79$ showed significant contributions in discriminating the effect of exercise for all groups. In addition to these ions, $m / z 43$ is contributing for CT and T1DM groups, $m / z, 73$ only for T1DM and $m / z, 83$ for T2DM. This indicates that these ions are strongly involved in forming a multivariate model to discriminate the effect of exercise in the three groups of subjects.

To evaluate the effect of exercise over the course of the 4 days, M-PLS-DA was used to classify between before and after exercise on each of the days. Four different models were made, and their performances are shown in Table 1(b).

Discrimination of before and after exercise was significantly relevant for all days, except for day 2 . The ability to discriminate was greatest for day 4 , followed by day 3 . This shows that the perceived metabolic effect of exercise increases from day to day, and is especially high on the last 2 days of the marches. The significance of the variable contribution is shown in a radar plot in Fig. 2.

The ions at $m / z 61,62$ and 79 are contributing significantly to the discriminative model on all 4 days. Other ions exhibit significance on some but not all days and these are summarized in Table 2. The possible compounds/ fragments for these ions measured in urine headspace are shown also in Table 2 with reference to the previously reported urine analysis and PTR-MS ion identification techniques.

The signals at $m / \mathrm{z} 61,62$ and 79 were consistently significant in both multivariate analyses. The signal observed at $m / z, 61$ showed a correlation with $m / z, 62$ (data not shown), relating to its ${ }^{13} \mathrm{C}(1.1 \%)$ carbon isotope with a slope value of $2.6 \%$; which is close to the theoretically predicted $2.3 \%$ for a two carbon ion. This strongly suggests that $m / z, 62$ is due to the ${ }^{13} \mathrm{C}$ isotope of $\mathrm{m} / \mathrm{z}, 61$ and that $m / z 61$ contains two carbon atoms. The ion signal observed at $m / z$ 79, was found to have a significant contribution for prolonged exercise. The signal of $\mathrm{m} / \mathrm{z}, 79$ was higher after exercise than before on day 2, 3 and 4. The correlation between $m / z, 61$ and $m / z, 79$ is $\mathrm{R}^{2}=0.88$. Therefore, $m / z 79$ may be the single hydrate cluster of $m / z \quad 61$ $(61+18=79)$. These correlations, along with knowledge of PTR-MS product ion identification imply that $\mathrm{m} / \mathrm{z} 61$, $m / z, 62$ and $m / z, 79$ are from the same compound. We 
Fig. 1 Radar plot per group showing the variable $(\mathrm{m} / \mathrm{z})$ contribution to the M-PLS-DA model classifying between before and after exercise

\section{Radarplot per group (all days)}

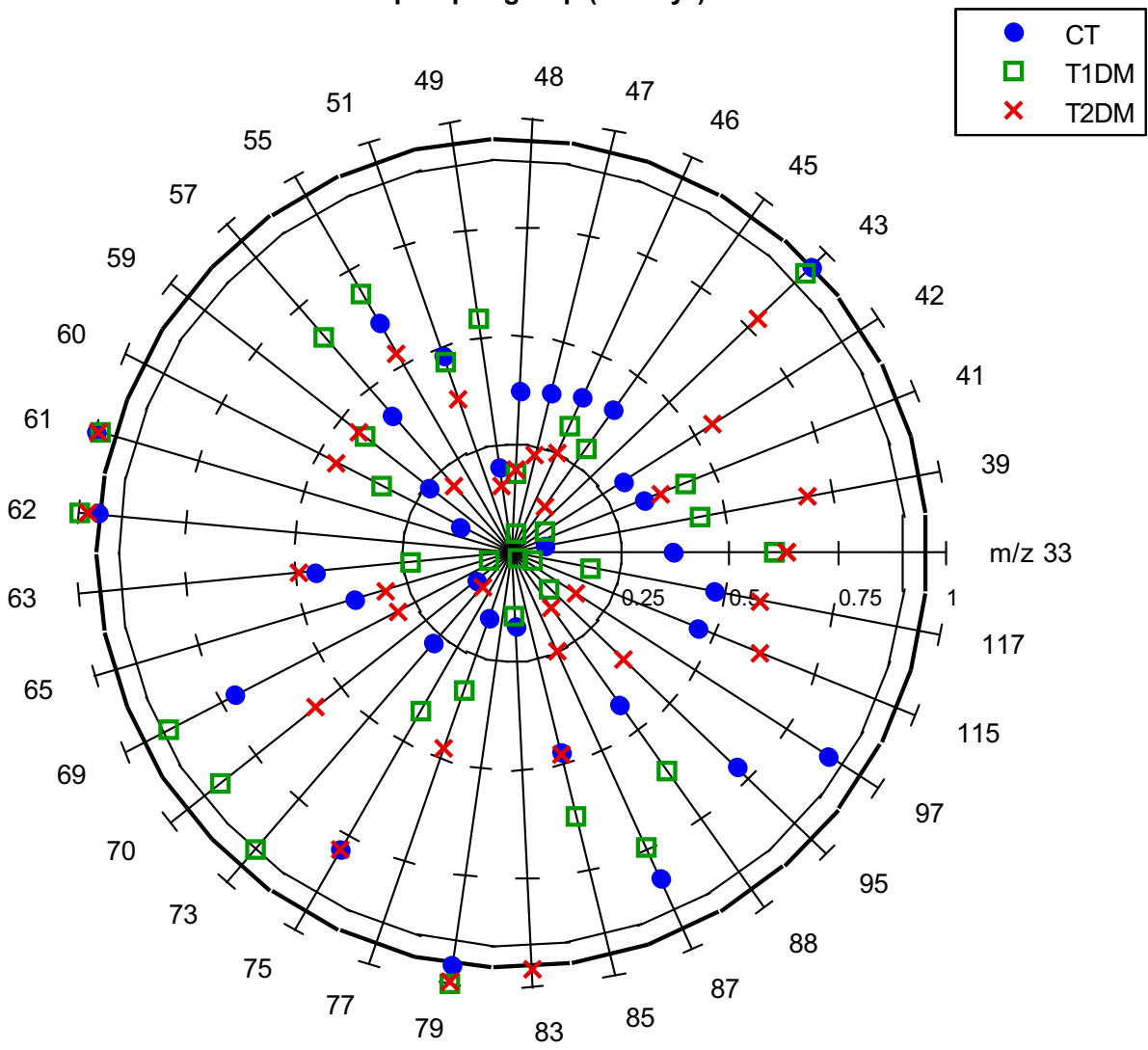

propose that they are all product ions of acetic acid, an identification that we base on putative annotation, (level 2) as described by Sumner et al. (2007). Using this identification, no additional information is provided by treating these ions separately. Therefore, univariate analysis of acetic acid is shown from here on by univariate analysis of the ion at $\mathrm{m} /$ $z 61$ and the effect of exercise on this ion is verified.

\subsection{Univariate analysis of acetic acid}

The multivariate analysis showed that acetic acid significantly contributed to the models and allowed for the effect of prolonged exercise to be discriminated. Therefore, this ion was analyzed by univariate analysis. In Fig. 3, box plots are shown for acetic acid for all the 4 days, including all subjects. There is a significant effect due to exercise on acetic acid for all days; with $p$-values, 0.03 on day 1 and $<0.001$ for days 2-4. Both univariate and multivariate analysis highlighted the significance of this ion with respect to an effect of exercise, therefore it is confirmed that this ion is a possible marker for the effect of exercise in urine headspace.

Apart from these ions, other ions were putatively annotated (level 2) (Sumner et al. 2007) based on previous identification in similar studies shown in Table 2; measured urine VOCs as an effect of exercise (Enea et al.
2010) or diabetic markers (Deja et al. 2013) or markers for other disorders (Huang et al. 2013; Troccaz et al. 2013).

In addition to the acetic acid signal, significant ions are observed that may be product ions of hexanoic acid. However the signal of either product ion or fragment ions didn't appear on each day in every group. This could be due to other ionic species being present at the same $m / z$ values.

\subsection{Univariate analysis of acetone $(\mathrm{m} / z \mathbf{5 9})$}

Wang et al. (2008) reported finding an equilibration between urine acetone and breath acetone in two subjects, implying a correlative relationship. In the present study, breath concentrations and urinary headspace concentrations of acetone were checked for correlation, but no correlation was observed. A strong correlation for acetone concentration in urinary headspace with exercise was reported by Orhan et al. (2004). Acetone is obviously detected using PTR-MS, with a product ion observed at $m / z$ 59. In the left panel of Fig. 4, the effect of exercise is shown on all participants with respect to the variation of acetone levels before and after exercise for the 4 consecutive days. For day 1 and day 2 there is no significant effect of exercise on measured headspace acetone $(p=0.46 ; p=0.33)$. However, on the last 2 days, day 3 and day 4 the effect due to prolonged exercise is significant, with 
Fig. 2 Radar plot per day showing the variable $(\mathrm{m} / \mathrm{z})$ contribution to the M-PLS-DA model classifying between before and after exercise

\section{Radarplot per day (all groups)}

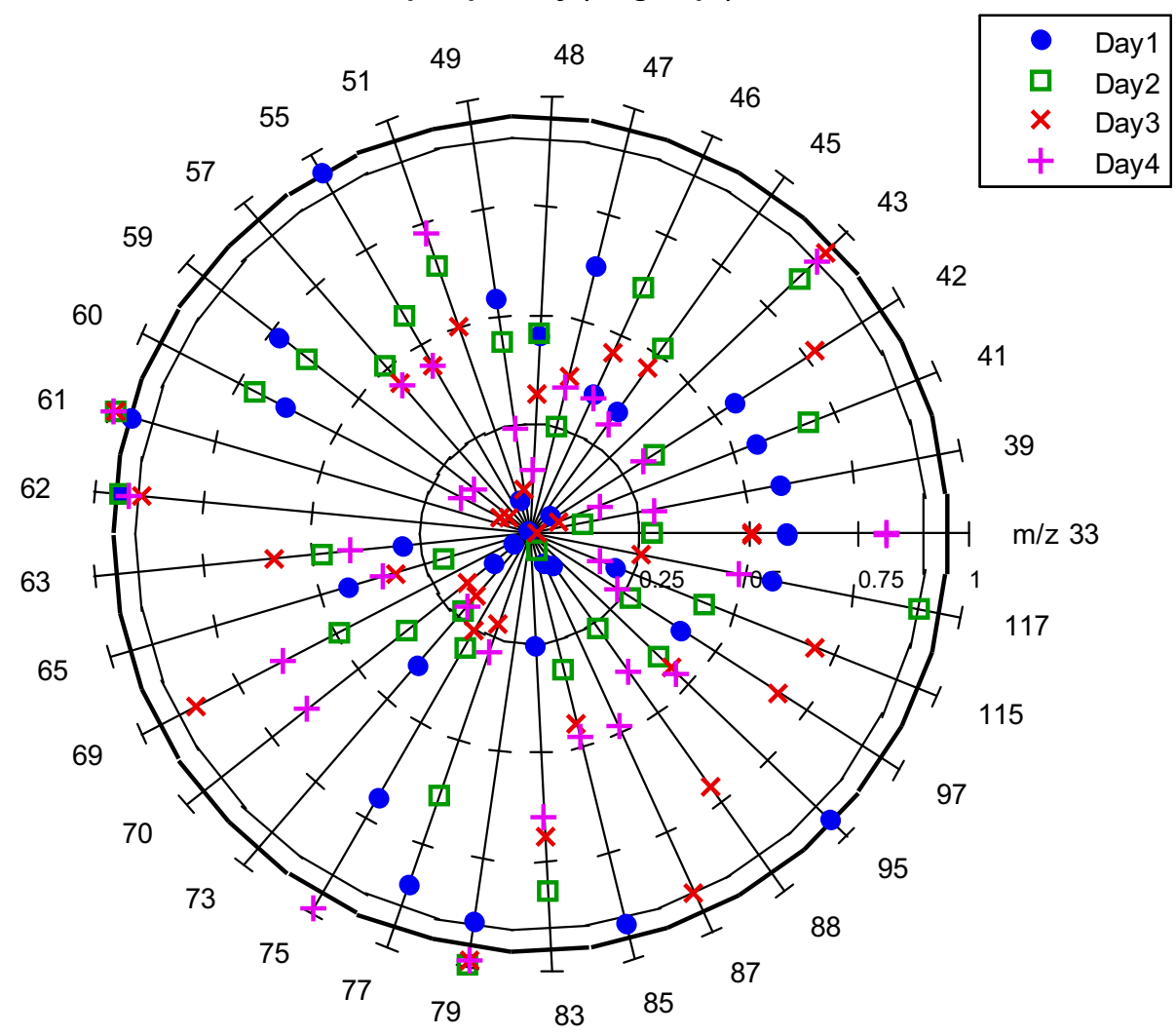

$p$-values 0.049 and $<0.001$ respectively. The effect of exercise on CT subjects is significant $(p<0.001)$, but not on T1DM and T2DM subjects (Fig. 4, right panel). In contrast to our previous experiments with breath acetone (Samudrala et al. 2014); there is a significant, exercise effect on acetone concentration in urine headspace when judged as a function of time.

\section{Discussion}

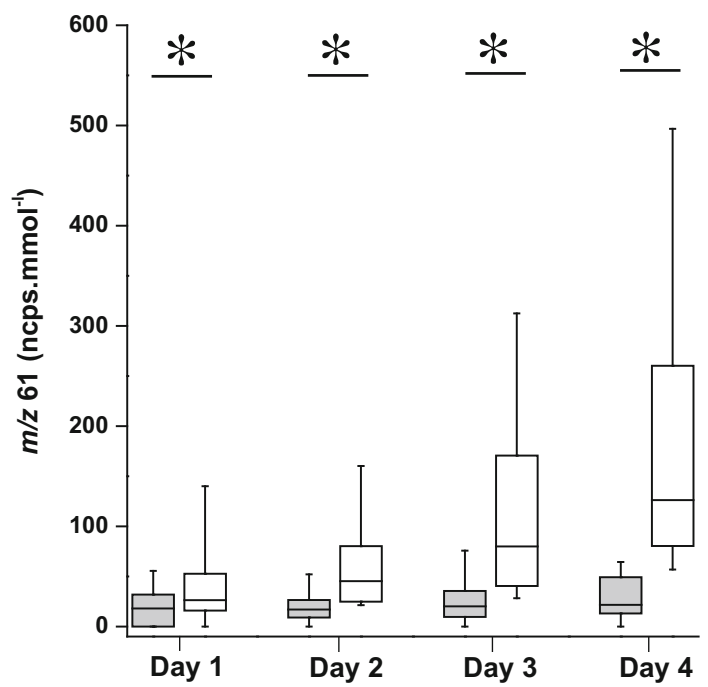

Fig. 3 Urine headspace $m / z, 61$ concentrations measured before and after exercise over 4 consecutive days for all participants. Data are displayed as box plots showing the median, interquartile ranges (25, $75 \%$ ); whiskers indicate the $10-90 \%$ values. *indicates $p<0.05$
Multivariate analysis revealed which measured ion signals contributed significantly to discriminating the effect of exercise. These results are validated using cross-validation and permutation testing, confirming the validity of the analysis for this data. The low number of samples is not optimal, and the results of the multivariate analysis should be confirmed in a larger cohort. These ion signals are shown in Table 2 with putative annotations. In PTR-MS, identification of ions is difficult and there is no library for the compound identification. However, tentative identification can be made depending on an ion's fragmentation pattern and/or using isotopic ratios (Crespo et al. 2012) or comparing with the NIST mass spectrum (NIST 2011).

Acetate is a product of fatty acid metabolism. It can either convert into acetyl-CoA and participate in the Krebs cycle to produce energy, or with two molecules of acetate it can form acetoacetate which further converts into acetone due to decarboxylation (Miekisch et al. 2004). Xu et al. (2014) have recently raised the level of interest in acetate 
Table 2 Ions from the headspace of urine measurements that showed significant contribution in discriminating the effect of the exercise during the 4 days walking program

\begin{tabular}{|c|c|c|c|c|c|}
\hline$m / z$ & $\begin{array}{l}\text { Possible protonated } \\
\text {-monomer (M) } \\
\text {-fragment (F) } \\
\text {-cluster (C) } \\
\text {-isotope (I) }\end{array}$ & $\begin{array}{l}\text { Effect of exercise } \\
\text { on each group (Fig. 2) } \\
\text { CT/T1DM/T2DM }\end{array}$ & $\begin{array}{l}\text { Effect of exercise } \\
\text { on each day (Fig. 3) } \\
\text { Day 1/Day 2/Day 3/ } \\
\text { Day } 4\end{array}$ & $\begin{array}{l}\text { Inter-comparison } \\
\text { with previous } \\
\text { related NMR and } \\
\text { MS studies }\end{array}$ & $\begin{array}{l}\text { Volatile } \\
\text { compound } \\
\text { identification }^{\text {a }}\end{array}$ \\
\hline 43 & Acetic acid; CAS: 64-19-7 (F) & CT, T1DM & Day 3, Day 4 & Enea et al. (2010) & NIST-MS*; FCA \\
\hline 55 & $\begin{array}{l}\text { Water cluster }(\mathrm{C}) \\
\text { Hexanoic acid; CAS: } \\
\quad \text { 142-62-1 (F) } \\
\text { Phenol; CAS: 108-95-2 (F) }\end{array}$ & & Day 1 & $\begin{array}{l}\text { Brown et al. (2010), } \\
\text { Huang et al. (2013), } \\
\text { Troccaz et al. (2013) }\end{array}$ & NIST-MS* \\
\hline $61,62,79$ & Acetic acid (M, I, C) & CT, T1DM, T2DM & $\begin{array}{l}\text { Day } 1 \text {, Day } 2 \text {, } \\
\text { Day } 3 \text {, Day } 4\end{array}$ & Enea et al. (2010) & ICA; CCA \\
\hline 73 & $\begin{array}{l}\text { Succinic acid; CAS: } \\
\text { 110-15-6 (F) } \\
\text { Hexanoic acid }(\mathrm{F})\end{array}$ & T1DM & & $\begin{array}{l}\text { Deja et al. (2013), } \\
\text { Huang et al. (2013) }\end{array}$ & NIST-MS*; CID \\
\hline 75 & $\begin{array}{l}\text { Methional; } C A S: \\
3268-49-3(\mathrm{~F})\end{array}$ & & Day 4 & Troccaz et al. (2013) & NIST-MS* \\
\hline 83 & Hexanoic acid $(\mathrm{F})$ & $\mathrm{T} 2 \mathrm{DM}$ & & Huang et al. (2013) & NIST-MS*; CID \\
\hline 85 & $\begin{array}{l}\text { 3-Hydroxy butyric acid; } \\
C A S: \text { 300-85-6 (F) } \\
\text { Succinic acid (F) }\end{array}$ & & Day 1 & Deja et al. (2013) & NIST-MS* \\
\hline 87 & $\begin{array}{l}\text { 3-Hydroxy butyric acid (F) } \\
\text { Hexanoic acid (F) }\end{array}$ & & Day 3 & $\begin{array}{l}\text { Deja et al. (2013), } \\
\text { Huang et al. (2013) }\end{array}$ & NIST-MS*; CID \\
\hline 95 & $\begin{array}{l}\text { Dimethyl disulfide; } \\
\text { CAS: 624-92-0 (M) }\end{array}$ & & Day1 & Troccaz et al. (2013) & NIST-MS* \\
\hline 117 & Hexanoic acid (M) & & Day2 & Huang et al. (2013) & NIST-MS*; CID \\
\hline
\end{tabular}

Possible identification of these ions in correspondence with the related literature and by some additional identification methods

Star $(*)$ represents that the ion is tentatively identified

${ }^{\text {a }}$ NIST-MS, Identification by comparison with NIST mass spectrum (NIST 2011); ICA, identification by isotopic correlation analysis; CCA, cluster correlation analysis, using clusters formed due to the reaction with water; FCA, fragment correlation analysis, correlation between potential fragment and monomer ions; CID, suggested fragments from experiments performed by Ion trap mass spectrometer with the pure compound

in relation to training by showing its importance in the mammalian stress-response to hypoxia. In addition, Fushimi et al. (Fushimi and Sato 2005; Fushimi et al. 2001) have shown that increased dietary acetic acid can stimulate glycogenesis, leading to increased glycogen recovery in skeletal muscles. Going on to conclude that increased dietary intake of acetic acid may be beneficial in glycogen recovery post-exercise.

In this study, evidence is presented that acetate in urine may be used to monitor the effect of subsequent days intense walking. Using breath analysis, of the same subjects, acetone was observed as a direct reflection of burning fat as an effect of walking (Samudrala et al. 2014). In univariate analysis, it is clearly evidenced that for each of the 4 days of exercise, acetate levels in urine are raised by the exercise, similar to the acetone behavior in breath analysis of the same cohort.

Two other ions, $m / \mathrm{z} 43$ and $m / z 79$ could be the fragment and monohydrate cluster of acetic acid, drawing on evidence from the NIST chemistry web book and correlation analysis. As mentioned earlier, ketone bodies can be detected in urine samples. One of the ketone bodies that has been shown by Samudrala et al. (2014) to have a correlation with breath acetone is BOHB. In the present study, $m / z, 83$ and $m / z, 87$ showed contribution to the model on day 1 and day 3 , respectively. These could be ion fragments of protonated BOHB in comparison with NIST mass spectrum. Previous studies also showed the occurrence of this compound in the urine of diabetic subjects (Deja et al. 2013) and due to physical exercise (Enea et al. 2010). Since these two ions are tentatively identified in this study, there is a possibility that several other compounds can have the same ion as a fragment. However, the ratio of these fragment ions will vary depending on the parent ion. Protonated hexanoic acid $(\mathrm{m} / z, 117)$ has a major fragment ion at $m / z, 87$. There are also minor fragment ion peaks at $m / z 73, m / z, 83$ and $m / z$ 55. Though these ions were highlighted on different days in different groups of people there 


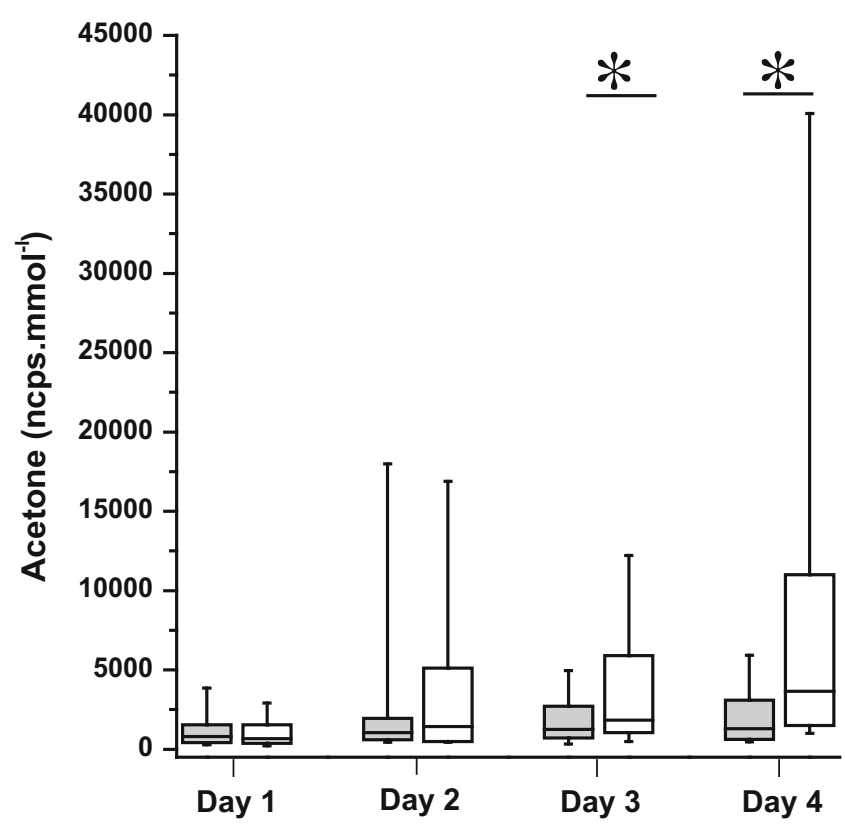

Fig. 4 Univariate analysis of acetone: Left panel Urine headspace acetone concentrations measured before and after the walk over 4 consecutive days for all participants. Right panel Headspace acetone concentrations measured before and after the walk in CT, T1DM, and

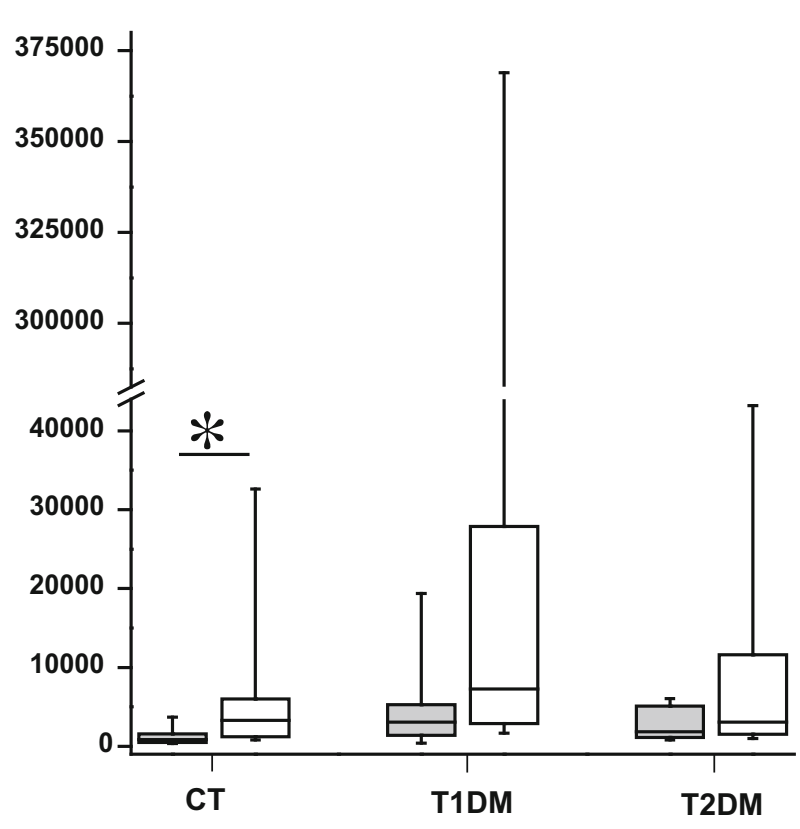

T2DM for 4 days. Data are displayed as box plots showing the median, interquartile ranges $(25 \%, 75 \%)$; whiskers indicate the $10-90 \%$ values. *indicates $p<0.05$

hexanoic acids seen in urine. That a link exists between acetate, hexanoate and lactate is also of note because lactic acid is a commonly used marker for muscular activity (Gladden 2004). A correlation was looked for between hexanoic acid and acetic acid in this study, however no such correlation was found.

Urine is an odorous biological liquid. Therefore, the presence of odor compounds like sulfides are possible, due to the presence of different bacteria. A recent study by Troccaz et al. (2013) showed that dimethyl sulfide, trimethylamine, dimethyl disulfide, methional, and some phenols are responsible for this odor due to several bacterial interactions. However, the presence of these bacteria may vary from person to person, due to the changes in body conditions and the amount of by-products the body is producing for excretion. This is the reason that methional and dimethyl disulphide are mentioned in Table 2 as possible compounds.

Urine analysis is a non-invasive method for investigating integrated responses after application of stress/exercise on humans (Enea et al. 2010). However, only a few studies have reported the impact of physical exercise on urinary headspace VOCs (Orhan et al. 2004; Pechlivanis et al. 2010; Enea et al. 2010), while others report the effects of physical characters such as gender, age, and diurnal variation (Slupsky et al. 2007). A recent study on urinary headspace by Enea et al. showed that metabolomics is a plausible explanation for the increase in acetic and 
promising tool to gain insights into changes induced by short term, intense physical exercise, with measurements made using proton NMR spectroscopy (Enea et al. 2010). In their study they observed the changes in the creatinine, lactate, pyruvate, acetate, BOHB and hypoxanthine due to the effect of short term intensive exercise.

This study represents the first measurements of the headspace of urine samples using PTR-MS to investigate the effect of prolonged exercise. Pinggera et al. (2005) have used PTR-MS for urinary measurements of acetonitrile in smokers, although they did not look for correlation with breath acetonitrile; they did find correlation between acetonitrile and smoking behavior. Using SIFT-MS, Wang et al. (2008) claimed that acetone was equilibrated amongst the body fluids by comparing acetone in breath with urinary headspace measurements of healthy volunteers. This claim is made on only a very limited cohort size of two volunteers. The data which we present did not show a correlation between urinary acetone and breath acetone.

Metabolites produced in the body as a result of different metabolisms are diffused from blood into urine and breath for further elimination. Breathing rate is typically $15-20$ times per minute for adults and urination frequency is typically 6-7 times per $24 \mathrm{~h}$ (Nitti 2002). This variation in timing means that breath analysis provides insight into metabolic changes at the moment the sample is taken, whereas metabolites in urine samples result from a longer term diffusion process. This is the expected reason for the difference in the behavior of urinary acetone and breath acetone as an effect of prolonged exercise.

Samples of urine collected by this study were not treated with any antimicrobial agent, such as sodium azide. Samples were also not immediately deep-frozen. For these reasons, the notion that bacterial growth or metabolic degradation may have occurred in the sample and could have contributed compounds to the VOC profile cannot be discounted. In future studies it is recommended to add an antibacterial agent (Orhan et al. 2004; Slupsky et al. 2007) and to deep-freeze samples immediately after acquisition, as suggested in a review article by Want et al. (2010). Typical inter-day and intra-day variations of urine headspace samples measured with PTR-MS were not evaluated for this study. Diurnal variation has been analyzed by Slupsky et al. (2007) using NMR spectroscopy. A careful understanding of biological variation under control conditions is useful for assessing the importance of observed variation in non-control groups.

Previous studies reported mass spectrometry based techniques in measuring urine headspace measurements for different applications (Smith et al. 1999; Wahl et al. 1999; Huang et al. 2013). However, most of the studies use NMR spectroscopy to analyze urine samples. Metabolomics is a rapidly expanding field in recent years, due in part to the advancement of NMR. This technique detects a wide range of compounds with easy, straightforward quantification. However, NMR has a limited sensitivity that can detect only concentrations of $10 \mu \mathrm{M}$, or a few nmol, at high fields using cryoprobes (Pan and Raftery 2007). Furthermore, the time to record simple spectra can be $4-5 \mathrm{~min}$, whereas PTR-MS has the capability to measure in a matter of seconds, and while in this study measurements were performed over a few minutes, with the implementation of auto-sampling methodology samples could be measured much faster.

PTR-MS has shown itself capable of revealing considerable shifts in the expression of several urine volatiles. These shifts could be analyzed very rapidly. Fast analyses could be coupled to dedicated multivariate statistical methods, which have already proved their merits in metabolomics, and do so again in this study. Thereby, this technology could be employed in large-scale public events. Specifically those events which focus on diet and physical exercise and in which a large number of individuals can be monitored non-invasively within a time-frame that would allow variations in their physiological states to be observed.

\section{Concluding remarks}

Multivariate analysis allowed for discrimination of before and after exercise for all three groups and on three out of 4 days. The advantage of using multivariate analysis in highlighting 12 ions from 33 is shown clearly by this study. Acetic acid in urinary headspace is identified as a significant marker for exercise effects induced by walking. An increase in acetic acid is observed after exercise for all days, and all groups. The potential to use acetic acid in urine to monitor exercise effects is exhibited and may have particular application in conjunction with PTR-MS for monitoring the effect on participants in a mass participation exercise event. Analysis of acetone concentration with univariate tools revealed different information when compared to breath as a function of exercise; making known an interesting effect of time over the 4 days. Breath samples provide insight into metabolic changes at the moment the sample is taken, whereas metabolites in urine samples result from a longer term diffusion process. This difference in timing may allow complementary information to be obtained.

Acknowledgments The authors wish to acknowledge the Department of Physiology at Radboud University Medical Centre and Luc Tappy from University of Lausanne for useful discussions. The authors also thank the organizers of the International Four Days Marches from Nijmegen and the volunteers who participated in this study.

Conflict of interest Each author declares no conflict of interest. 
Funding The authors acknowledge funding from the European Regional Development Fund, province of Gelderland; Project: GOEFRO; 2009-010034. Additional funding was provided by the Netherlands Organisation for Scientific Research (NWO) in the framework of the Technology Area COAST; Project: Breath Volatiles; 053.21.103.

Compliance with ethical requirements This study was approved by the Medical Ethical Committee of the Radboud University Nijmegen Medical Centre. Informed consent was obtained from all individual participants included in the study, and the study was conducted in accordance with the Declaration of Helsinki.

Open Access This article is distributed under the terms of the Creative Commons Attribution 4.0 International License (http://creativecommons.org/licenses/by/4.0/), which permits unrestricted use, distribution, and reproduction in any medium, provided you give appropriate credit to the original author(s) and the source, provide a link to the Creative Commons license, and indicate if changes were made.

\section{References}

Amann, A., \& Smith, D. (2013). Volatile biomarkers: Non-invasive diagnosis in physiology and medicine. Amsterdam: Elsevier Science.

Barnes, P. J., Dweik, R. A., Gelb, A. F., Gibson, P. G., George, S. C., Grasemann, H., et al. (2010). Exhaled nitric oxide in pulmonary diseases: A comprehensive review. Chest, 138(3), 682-692. doi:10.1378/chest.09-2090.

Blake, R. S., Monks, P. S., \& Ellis, A. M. (2009). Proton-transfer reaction mass spectrometry. Chemical Reviews, 109(3), 861-896. doi:10.1021/cr800364q.

Brown, P., Watts, P., Mark, T. D., \& Mayhew, C. A. (2010). Proton transfer reaction mass spectrometry investigations on the effects of reduced electric field and reagent ion internal energy on product ion branching ratios for a series of saturated alcohols. International Journal of Mass Spectrometry, 294(2-3), 103-111. doi:10.1016/j.ijms.2010.05.028.

Buszewski, B., Kęsy, M., Ligor, T., \& Amann, A. (2007). Human exhaled air analytics: biomarkers of diseases. Biomedical Chromatography, 21(6), 553-566. doi:10.1002/bmc.835.

Chen, R., Mias, George I., Li-Pook-Than, J., Jiang, L., Lam, Hugo Y. K., Chen, R., et al. (2012). Personal omics profiling reveals dynamic molecular and medical phenotypes. Cell, 148(6), 1293-1307. doi:10.1016/j.cell.2012.02.009.

Costello, B., Amann, D. L., Al-Kateb, A., Flynn, H., Filipiak, D. L., Khalid, T., et al. (2014). A review of the volatiles from the healthy human body. Journal of Breath Research, 8(1), 014001.

Crespo, E., Hordijk, C. A., de Graaf, R. M., Samudrala, D., Cristescu, S. M., Harren, F. J., et al. (2012). On-line detection of rootinduced volatiles in Brassica nigra plants infested with Delia radicum L. root fly larvae. Phytochemistry, 84, 68-77. doi:10. 1016/j.phytochem.2012.08.013.

Deja, S., Barg, E., Mlynarz, P., Basiak, A., \& Willak-Janc, E. (2013). H-1 NMR-based metabolomics studies of urine reveal differences between type 1 diabetic patients with high and low $\mathrm{HbAc1}$ values. Journal of Pharmaceutical and Biomedical Analysis, 83, 43-48. doi:10.1016/j.jpba.2013.04.017.

Enea, C., Seguin, F., Petitpas-Mulliez, J., Boildieu, N., Boisseau, N., Delpech, N., et al. (2010). (1)H NMR-based metabolomics approach for exploring urinary metabolome modifications after acute and chronic physical exercise. Analytical and
Bioanalytical Chemistry, 396(3), 1167-1176. doi:10.1007/ s00216-009-3289-4.

Fushimi, T., \& Sato, Y. (2005). Effect of acetic acid feeding on the circadian changes in glycogen and metabolites of glucose and lipid in liver and skeletal muscle of rats. British Journal of Nutrition, 94(5), 714-719. doi:10.1079/bjn20051545.

Fushimi, T., Tayama, K., Fukaya, M., Kitakoshi, K., Nakai, N., Tsukamoto, Y., et al. (2001). Acetic acid feeding enhances glycogen repletion in liver and skeletal muscle of rats. Journal of Nutrition, 131(7), 1973-1977.

Gladden, L. B. (2004). Lactate metabolism: A new paradigm for the third millennium. The Journal of Physiology, 558(1), 5-30. doi:10.1113/jphysiol.2003.058701.

Herbig, J., Muller, M., Schallhart, S., Titzmann, T., Graus, M., \& Hansel, A. (2009). On-line breath analysis with PTR-TOF. Journal of Breath Research, 2, 027004.

Huang, J. Z., Kumar, S., Abbassi-Ghadi, N., Spanel, P., Smith, D., \& Hanna, G. B. (2013). Selected ion flow tube mass spectrometry analysis of volatile metabolites in urine headspace for the profiling of gastro-esophageal cancer. Analytical Chemistry, 85(6), 3409-3416. doi:10.1021/ac4000656.

Januszewski, A. S., Jenkins, A. J., Baynes, J. W., \& Thorpe, S. R. (2005). Lipid-derived modifications of plasma proteins in experimental and human diabetes. Annals of the New York Academy of Sciences, 1043(1), 404-412. doi:10.1196/annals. 1333.047.

Lindinger, W., Hansel, A., \& Jordan, A. (1998). On-line monitoring of volatile organic compounds at pptv levels by means of ProtonTransfer-Reaction Mass Spectrometry (PTR-MS) Medical applications, food control and environmental research. International Journal of Mass Spectrometry and Ion Processes, 173, 191-241.

Lourenço, C., \& Turner, C. (2014). Breath analysis in disease diagnosis: Methodological considerations and applications. Metabolites, 4(2), 465-498.

Mazzatenta, A., Di Giulio, C., \& Pokorski, M. (2013). Pathologies currently identified by exhaled biomarkers. Respiratory Physiology \& Neurobiology, 187(1), 128-134. doi:10.1016/j.resp.2013. 02.016 .

Miekisch, W., Schubert, J. K., \& Noeldge-Schomburg, G. F. (2004). Diagnostic potential of breath analysis-focus on volatile organic compounds. Clinica Chimica Acta, 347(1-2), 25-39. doi:10.1016/j.cccn.2004.04.023.

Mochalski, P., Krapf, K., Ager, C., Wiesenhofer, H., Agapiou, A., Statheropoulos, M., et al. (2012). Temporal profiling of human urine VOCs and its potential role under the ruins of collapsed buildings. Toxicology Mechanisms and Methods, 22(7), 502-511. doi: 10.3109/15376516.2012.682664

NIST Chemistry Data WebBook (2011). U.S. Secretary of Commerce on behalf of the United States of America. http://webbook.nist. gov/chemistry/.

Nitti, V. W. (2002). Clinical impact of overactive bladder. Reviews in Urology, 4(Suppl 4), S2-S6.

Opitz, D., Lenzen, E., Schiffer, T., Hermann, R., Hellmich, M., Bloch, W., et al. (2014). Endurance training alters skeletal muscle MCT contents in T2DM men. International Journal of Sports Medicine, 35(13), 1065-1071. doi:10.1055/s-00341371838.

Orhan, H., Van Holland, B., Krab, B., Moeken, J., Vermeulen, N. P. E., Hollander, P., et al. (2004). Evaluation of a multiparameter biomarker set for oxidative damage in man: Increased urinary excretion of lipid, protein and DNA oxidation products after one hour of exercise. Free Radical Research, 38(12), 1269-1279. doi:10.1080/10715760400013763.

Pan, Z. Z., \& Raftery, D. (2007). Comparing and combining NMR spectroscopy and mass spectrometry in metabolomics. 
Analytical and Bioanalytical Chemistry, 387(2), 525-527. doi:10.1007/s00216-006-0687-8.

Pechlivanis, A., Kostidis, S., Saraslanidis, P., Petridou, A., Tsalis, G., Mougios, V., et al. (2010). H-1 NMR-based metabonomic investigation of the effect of two different exercise sessions on the metabolic fingerprint of human urine. Journal of Proteome Research, 9(12), 6405-6416. doi:10.1021/Pr100684t.

Pinggera, G. M., Lirk, P., Bodogri, F., Herwig, R., Steckel-Berger, G., Bartsch, G., et al. (2005). Urinary acetonitrile concentrations correlate with recent smoking behaviour. BJU International, 95(3), 306-309. doi:10.1111/j.1464-410X.2005.05288.x.

Roukos, D. H. (2008). Innovative genomic-based model for personalized treatment of gastric cancer: Integrating current standards and new technologies. Expert Review of Molecular Diagnostics, 8(1), 29-39. doi:10.1586/14737159.8.1.29.

Saary, M. J. (2008). Radar plots: A useful way for presenting multivariate health care data. Journal of Clinical Epidemiology, 61(4), 311-317. doi:10.1016/j.jclinepi.2007.04.021.

Samudrala, D., Lammers, G., Mandon, J., Blanchet, L., Schreuder, T. H. A., Hopman, M. T., et al. (2014). Breath acetone to monitor life style interventions in field conditions: An exploratory study. Obesity, 22(4), 980-983. doi:10.1002/oby.20696.

Schwarz, K., Filipiak, W., \& Amann, A. (2009). Determining concentration patterns of volatile compounds in exhaled breath by PTR-MS. Journal of Breath Research, 2, 027002.

Slupsky, C. M., Rankin, K. N., Wagner, J., Fu, H., Chang, D., Weljie, A. M., et al. (2007). Investigations of the effects of gender, diurnal variation, and age in human urinary metabolomic profiles. Analytical Chemistry, 79(18), 6995-7004. doi:10. 1021/Ac0708588.

Smit, S., van Breemen, M. J., Hoefsloot, H. C. J., Smilde, A. K., Aerts, J. M. F. G., \& de Koster, C. G. (2007). Assessing the statistical validity of proteomics based biomarkers. Analytica Chimica Acta, 592(2), 210-217. doi:10.1016/j.aca.2007.04.043.

Smith, D., Spanel, P., Holland, T. A., Al Singari, W., \& Elder, J. B. (1999). Selected ion flow tube mass spectrometry of urine headspace. Rapid Communications in Mass Spectrometry, 13(8), 724-729. doi:10.1002/(Sici)1097-0231(19990430)13:8<724.

Smolinska, A., Klaassen, E. M., Dallinga, J. W., van de Kant, K. D., Jobsis, Q., Moonen, E. J., et al. (2014). Profiling of volatile organic compounds in exhaled breath as a strategy to find early predictive signatures of asthma in children. PLoS One, 9(4), e95668. doi:10.1371/journal.pone.0095668.

Steeghs, M., Bais, H. P., de Gouw, J., Goldan, P., Kuster, W., Northway, M., et al. (2004). Proton-transfer-reaction mass spectrometry as a new tool for real time analysis of rootsecreted volatile organic compounds in arabidopsis. Plant Physiology, 135(1), 47-58. doi:10.1104/pp.104.038703.

Storer, M., Dummer, J., Lunt, H., Scotter, J., McCartin, F., Cook, J., et al. (2011). Measurement of breath acetone concentrations by selected ion flow tube mass spectrometry in type 2 diabetes. Journal of Breath Research, 5(4), 046011.
Sumner, L. W., Amberg, A., Barrett, D., Beale, M. H., Beger, R., Daykin, C. A., et al. (2007). Proposed minimum reporting standards for chemical analysis. Metabolomics, 3(3), 211-221. doi:10.1007/s11306-007-0082-2.

Szymańska, E., van Dorsten, F., Troost, J., Paliukhovich, I., van Velzen, E. J., Hendriks, M. W. B., et al. (2012). A lipidomic analysis approach to evaluate the response to cholesterollowering food intake. Metabolomics, 8(5), 894-906. doi:10. 1007/s11306-011-0384-2.

Troccaz, M., Niclass, Y., Anziani, P., \& Starkenmann, C. (2013). The influence of thermal reaction and microbial transformation on the odour of human urine. Flavour and Fragrance Journal, 28(4), 200-211. doi:10.1002/Ffj.3143.

Turner, C., Parekh, B., Walton, C., Španěl, P., Smith, D., \& Evans, M. (2008). An exploratory comparative study of volatile compounds in exhaled breath and emitted by skin using selected ion flow tube mass spectrometry. Rapid Communications in Mass Spectrometry, 22(4), 526-532. doi:10.1002/rcm.3402.

van Dam, N. M., Samudrala, D., Harren, F. J. M., \& Cristescu, S. M. (2012). Real-time analysis of sulfur-containing volatiles in Brassica plants infested with root-feeding Delia radicum larvae using proton-transfer reaction mass spectrometry. AoB Plants, 2012, pls021. doi:10.1093/aobpla/pls021.

van Velzen, E. J. J., Westerhuis, J. A., van Duynhoven, J. P. M., van Dorsten, F. A., Hoefsloot, H. C. J., Jacobs, D. M., et al. (2008). Multilevel data analysis of a crossover designed human nutritional intervention study. Journal of Proteome Research, 7(10), 4483-4491. doi:10.1021/pr800145j.

Wahl, H. G., Hoffmann, A., Luft, D., \& Liebich, H. M. (1999). Analysis of volatile organic compounds in human urine by headspace gas chromatography-mass spectrometry with a multipurpose sampler. Journal of Chromatography A, 847(1-2), 117-125. doi:10.1016/S0021-9673(99)00017-5.

Wang, T., Spanel, P., \& Smith, D. (2008). Selected ion flow tube mass spectrometry of 3-hydroxybutyric acid, acetone and other ketones in the headspace of aqueous solution and urine. International Journal of Mass Spectrometry, 272(1), 78-85. doi:10.1016/j.ijms.2008.01.002.

Want, E. J., Wilson, I. D., Gika, H., Theodoridis, G., Plumb, R. S., Shockcor, J., et al. (2010). Global metabolic profiling procedures for urine using UPLC-MS. Nature Protocols, 5(6), 1005-1018.

Westerhuis, J., Hoefsloot, H. J., Smit, S., Vis, D., Smilde, A., van Velzen, E. J., et al. (2008). Assessment of PLSDA cross validation. Metabolomics, 4(1), 81-89. doi:10.1007/s11306-0070099-6.

Xu, M., Nagati, J. S., Xie, J., Li, J. W., Walters, H., Moon, Y. A., et al. (2014). An acetate switch regulates stress erythropoiesis. Nature Medicine, 20(9), 1018-1026. doi:10.1038/nm.3587.

Zweig, M., \& Campbell, G. (1993). Receiver-operating characteristic (ROC) plots: A fundamental evalutation tool in clinical medicine. Clinical Chemistry, 39(4), 561-577. 